\title{
Quasi-Invariance of Measures on an Infinite Dimensional Vector Space and the Continuity of the Characteristic Functions
}

\author{
By \\ Yasuo YAMASAKI*
}

\section{Introduction}

Since the Bochner theorem was extended to the infinite dimensional case by Minlos [1] and Sazonov [2], the continuity of a characteristic function has been discussed mainly in connection with the carrier of the corresponding measure.

However, the study of the relation between the continuity of a characteristic function and the quasi-invariance of the corresponding measure has been rather neglected. In this paper we shall discuss this problem. Our main results are as follows.

Let $E$ be a vector space, $E^{\prime}$ be its algebraical dual space, $\mu$ be a finite measure on $E^{\prime}$, and $\chi$ be the characteristic function of $\mu$ defined on $E$. Consider the weakest vector topology on $E$ that makes $\chi$ continuous, and denote it with $\tau_{\mu}$.

Let $T_{\mu}$ be the set of all translations on $E^{\prime}$ under which $\mu$ is quasi-invariant. $T_{\mu}$ is regarded as a subset of $E^{\prime}$ by identifying any translation $x \rightarrow x+a$ on $E^{\prime}$ with $a$. Then we have the following

Theorem. (1) We have $T_{\mu} \subset E_{\mu}^{*}\left(\subset E^{\prime}\right)$, where $E_{\mu}^{*}$ is the topological dual space of $E$ with respect to $\tau_{\mu}$.

(2) Let $E_{\tau}^{*}$ be the topological dual space of $E$ with respect to a locally convex topology $\tau$ on $E$. Then, $E_{\tau}^{*} \subset T_{\mu}\left(\subset E^{\prime}\right)$ implies that $\tau$ is weaker than $\tau_{\mu}$

Especially, if $\mu$ is $E_{\tau}^{*}$-quasi-invariant and if $\chi$ is continuous in $\tau$, then we have $T_{\mu}=E_{\tau}^{*}$ and $\tau=\tau_{\mu}$.

Using these results, we can estimate $T_{\mu}$ from the continuity of $\chi$.

Received January 18, 1979.

* Research Institute for Mathematical Sciences, Kyoto University, Kyoto 606, Japan. 
Combining this with the relation between the continuity of $\chi$ and the carrier of $\mu$, we can establish the relation between the carrier and quasi-invariance of $\mu$. This fact implies various results. For instance, we can give another proof of the following results due to Xia Dao-Xing [3].

1. Let $X$ be a separable and metrizable locally convex vector space, and $Y(\subset X)$ be a complete metrizable topological vector space imbedded continuously in $X$. If some Borel measure on $X$ is $Y$-quasi-invariant, then $Y$ has a neighbourhood of 0 which is totally bounded in $X$. (\$5.)

2. Let $X$ be a Hilbert space, and $Y(\subset X)$ be another Hilbert space imbedded continuously in $X$. Then, there exists a $Y$-quasi-invariant measure on $X$ if and only if the canonical imbedding $Y \rightarrow X$ is of the HilbertSchmidt type. Especially, $Y$ must be separable even if $X$ is not. (\$6.)

\section{$\S 1$. Linearization of a Topology}

In this section, as a preliminary discussion, we shall explain a method to linearize a topology on a vector space.

Proposition 1.1. Let $E$ be a vector space, and suppose that $E$ becomes a topological additive group with respect to a topology $\tau$ on E. Furthermore, assume that $\tau$ satisfies the following condition;

$$
\forall x \in E, \quad \forall V \in \mathfrak{B}, \quad \exists \alpha_{0}>0, \quad|\alpha| \leqq \alpha_{0} \Longrightarrow \alpha x \in V,
$$

where $\mathfrak{B}$ is a fundamental system of neighbourhoods of 0 with respect to $\tau$.

Then, putting

$$
U_{V}=\bigcap_{|\alpha| \geqq 1}(\alpha V),
$$

we obtain a topology $\tau^{\prime}$ in which $\mathfrak{H}=\left\{U_{V}\right\}_{V \in \mathfrak{B}}$ is a fundamental system of neighbourhoods of 0 . The topology $\tau^{\prime}$ is the weakest topology that is stronger than $\tau$ and compatible with the linear structure of $E$.

Definition 1.1. The topology $\tau^{\prime}$ given in Proposition 1.1 is called the linearization of $\tau$.

Proof of Proposition 1.1. Suppose that a topology $\tau^{\prime \prime}$ on $E$ is stronger than $\tau$ and compatible with the linear structure of $E$. Then, for each $V \in \mathfrak{B}$, there exist $\alpha_{0}>0$ and a neighbourhood $U$ of 0 in $\tau^{\prime \prime}$ such that $|\alpha| \leqq \alpha_{0}$ implies $\alpha U \subset V$. Therefore we have $\alpha_{0} U \subset U_{V}$. From the continuity of scalar multiplication, 
$\alpha_{0} U$ is a neighbourhood of 0 in $\tau^{\prime \prime}$, so that $\tau^{\prime \prime}$ is stronger than $\tau^{\prime}$.

The topology $\tau^{\prime}$ is evidently stronger than $\tau$. Next, we shall show that $\tau^{\prime}$ is compatible with the linear structure of $E$.

Since the addition in $E$ is continuous in $\tau$, for each $V \in \mathfrak{B}$ there exists $V^{\prime} \in \mathfrak{B}$ such that $V^{\prime}+V^{\prime} \subset V$. This implies $U_{V^{\prime}}+U_{V^{\prime}} \subset U_{V}$. Therefore, if we define a fundamental system of neighbourhoods of $x \in E$ by $\left\{x+U_{V}\right\}_{V \in \mathfrak{B}}$ (actually the topology $\tau^{\prime}$ is defined in this way), the addition in $E$ becomes continuous, so that $E$ is a topological additive group with respect to $\tau^{\prime}$.

In order to prove the continuity of the scalar multiplication, it is sufficient to show the following three facts:

1) $\forall V \in \mathfrak{B}, \quad \exists \alpha_{0}>0, \quad \exists V_{0} \in \mathfrak{B}, \quad|\alpha| \leqq \alpha_{0} \Longrightarrow \alpha U_{V_{0}} \subset U_{V}$,

2) $\forall \alpha \neq 0, \quad \forall V \in \mathfrak{B}, \quad \exists V_{0} \in \mathfrak{B}, \quad \alpha U_{V_{0}} \subset U_{V}$,

3) $\forall x \in E, \quad \forall V \in \mathfrak{B}, \quad \exists \alpha_{0}>0, \quad|\alpha| \leqq \alpha_{0} \Longrightarrow \alpha x \in U_{V}$.

From (1.2), we have $\alpha U_{V}=\bigcap_{|\beta| \geqq 1}(\alpha \beta V)=\bigcap_{|\beta| \geqq|\alpha|}(\beta V)$, so that $|\alpha| \leqq 1$ implies $\alpha U_{V} \subset U_{V}$. Therefore the condition 1) is satisfied choosing $V_{0}=V$ and $\alpha_{0}=1$.

Next, suppose that a real number $\alpha \neq 0$ is given, and choose an integer $n$ such that $|\alpha|<n$. Since the addition in $E$ is continuous in $\tau$, for each $V \in \mathfrak{B}$, there exists $V_{n} \in \mathfrak{B}$ such that

$$
\underbrace{V_{n}+V_{n}+\cdots+V_{n}}_{n \text { terms }} \subset V
$$

Especially we have $n V_{n} \subset V$. Therefore we have

$$
U_{V}=\bigcap_{|\beta| \geqq 1}(\beta V) \supset \underset{|\beta| \geqq 1}{\bigcap}\left(n \beta V_{n}\right)=\bigcap_{|\beta| \geqq n}\left(\beta V_{n}\right) \supset \underset{|\beta| \geqq|\alpha|}{\bigcap}\left(\beta V_{n}\right)=\alpha U_{V_{n}} .
$$

This shows that the condition 2) is satisfied.

Lastly, the assumption (1.1) is equivalent with

$$
\forall x \in E, \quad \forall V \in \mathfrak{B}, \quad \exists \alpha_{0}>0, \quad \alpha_{0} x \in U_{V} .
$$

Then, $|\alpha| \leqq \alpha_{0}$ implies $\alpha x=\alpha \alpha_{0}^{-1}\left(\alpha_{0} x\right) \in \alpha \alpha_{0}^{-1} U_{V} \subset U_{V}$. Thus the fact 3) has been proved.

Q.E.D.

\section{§2. The Characteristic Topology}

Let $E$ be a vector space, $E^{\prime}$ be its algebraical dual space, and $\mathfrak{B}_{E}$ be the smallest $\sigma$-algebra of $E^{\prime}$ in which every element of $E$, regarded as a function on $E^{\prime}$, becomes measurable. For a probability measure $\mu$ on $\left(E^{\prime}, \mathfrak{B}_{E}\right)$, we shall 
define the characteristic topology on $E$ as follows.

Let $\chi(\xi)$ be the characteristic function of $\mu$.

Proposition 2.1. There exists the weakest topology on E that makes $\chi(\xi)$ continuous and compatible with the linear structure of $E$.

Proof. Since $\chi$ is a positive definite function, we have the inequality:

$$
|\chi(\xi+\eta)-\chi(\xi)| \leqq \sqrt{2|1-\chi(\eta)|} .
$$

Therefore, if we put

$$
V_{\varepsilon}=\{\xi \in E ;|1-\chi(\xi)| \leqq \varepsilon\},
$$

then $\xi \in V_{\varepsilon}$ and $\eta \in V_{\varepsilon}$ imply $\xi+\eta \in V_{\varepsilon+\sqrt{2 \varepsilon}}$. In other words we have

$$
V_{\varepsilon}+V_{\varepsilon} \subset V_{\varepsilon+\sqrt{2 \varepsilon}}
$$

Thus, if we define a fundamental system of neighbourhoods of $\xi \in E$ by $\left\{\xi+V_{\varepsilon}\right\}_{\varepsilon>0}, E$ becomes a topological group (not necessarily Hausdorff). Furthermore, on every finite dimensional subspace of $E, \chi$ is continuous with respect to the Euclid topology, so that we have

$$
\forall \xi \in E, \quad \forall \varepsilon>0, \quad \exists \alpha_{0}>0, \quad|\alpha| \leqq \alpha_{0} \Longrightarrow \alpha \xi \in V_{\varepsilon} .
$$

Consequently, from Proposition 1.1, we can consider the linearization of this topology. The linearized topology is evidently the requested one in our Proposition.

Q.E.D.

Definition 2.1. The topology mentioned in Proposition 2.1 is called the characteristic topology of $\mu$ and denoted with $\tau_{\mu}$.

In the topology $\tau_{\mu}$, a fundamental system of neighbourhoods of 0 is given by $\left\{U_{\varepsilon}\right\}_{\varepsilon}>0$, where

$$
U_{\varepsilon}=\bigcap_{|\alpha| \geqq 1}\left(\alpha V_{\varepsilon}\right)=\left\{\xi \in E ; \sup _{|\alpha| \leqq 1}|1-\chi(\alpha \xi)| \leqq \varepsilon\right\} .
$$

The topology $\tau_{\mu}$ is not necessarily Hausdorff. The condition $\xi \in \bigcap_{\varepsilon>0} U_{\varepsilon}$ is equivalent with $\chi(\alpha \xi)=1$ for $|\alpha| \leqq 1$. Therefore, from (2.1) we have $\chi(\alpha \xi)=1$ for every $\alpha \in \boldsymbol{R}^{1}$, so that we have $(\xi, x)=0$ for $\mu$-almost all $x$. In fact, $\tau_{\mu}$ is a Hausdorff topology on the factor space $E / M$, where

$$
M=\{\xi \in E ;(\xi, x)=0 \text { for } \mu \text {-almost all } x\} .
$$

Suppose that $E$ becomes a topological vector space with respect to a topology $\tau$. Then from the definition of $\tau_{\mu}$, we have 
Proposition 2.2. $\chi(\xi)$ is continuous in $\tau$, if and only if $\tau$ is stronger than $\tau_{\mu} \cdot$

\section{§3. The Topology of Measure Convergence}

In this section, we shall prove that the characteristic topology is identical with the topology of measure convergence. First, we shall explain the latter.

Let $\mu$ be a probability measure on a measurable space $(X, \mathfrak{B})$, and $\mathfrak{F}$ be the set of all $\mathfrak{B}$-measurable real-valued functions on $X$. $\mathfrak{F}$ forms a vector space.

Definition 3.1. A sequence $\left\{f_{n}\right\} \subset \mathbb{F}$ is said to converge to 0 in measure, if

$$
\forall \alpha>0, \quad \mu\left(\left\{x \in X ;\left|f_{n}(x)\right| \geqq \alpha\right\}\right) \rightarrow 0 \quad(n \rightarrow \infty) .
$$

Definition 3.2. Consider a topology on $\mathfrak{F}$ whose fundamental system of neighbourhoods of 0 is given by $\left\{U_{\alpha, \varepsilon}\right\}_{\alpha>0, \varepsilon}>0$, where

$$
U_{\alpha, \varepsilon}=\{f \in \mathfrak{\mho} ; \mu(\{x \in X ;|f(x)| \geqq \alpha\})<\varepsilon\} .
$$

This topology is called the topology of measure convergence.

A sequence $\left\{f_{n}\right\} \subset \mathfrak{F}$ converges to 0 in measure, if and only if it converges to 0 in the topology of measure convergence.

$U_{\alpha, \varepsilon}$ is increasing with respect to both $\alpha$ and $\varepsilon$, so that $\left\{U_{1 / n, 1 / n}\right\}_{n=1,2, \ldots}$ becomes a fundamental system of neighbourhoods of 0 .

$\mathcal{F}$ is a topological vector space with respect to the topology of measure convergence. First, $|f(x)+g(x)| \geqq \alpha+\beta$ implies $|f(x)| \geqq \alpha$ or $|g(x)| \geqq \beta$, so that we have $U_{\alpha, \varepsilon}+U_{\beta, \delta} \subset U_{\alpha+\beta, \varepsilon+\delta}$, hence the addition in $\mathbb{F}$ is continuous. Next, $|f(x)| \geqq \alpha$ is equivalent with $|c f(x)| \geqq|c| \alpha$, so that we have $c U_{\alpha, \varepsilon}=U_{|c| \alpha . \varepsilon^{\circ}}$ Combining this with the fact:

$$
\forall f \in \mathfrak{F}, \quad \forall \varepsilon>0, \quad \exists \alpha>0, \quad f \in U_{\alpha, \varepsilon},
$$

we see that the scalar multiplication in $\mathfrak{F}$ is also continuous.

The topology of measure convergence is not necessarily Hausdorff. The condition $f \in \underset{\alpha>0, \varepsilon>0}{\cap} U_{\alpha, \varepsilon}$ is equivalent with $f(x)=0$ for $\mu$-almost all $x$. This means that the topology of measure convergence is Hausdorff on the factor space $\mathfrak{F} / \mathfrak{M}$, where

$$
\mathfrak{M}=\{f \in \mathfrak{F} ; f(x)=0 \text { for } \mu \text {-almost all } x\} .
$$


Here, we shall give another representation of the topology of measure convergence.

Let $F(t)$ be a bounded continuous function on $[0, \infty)$. For $f \in \mathfrak{F}$, we shall put

$$
I_{F}(f)=\int_{X} F(|f(x)|) d \mu(x) .
$$

Lemma. 1) $I_{F}(f)$ is continuous at $f=0$ in the topology of measure convergence.

2) If we assume that

$$
\forall \alpha>0, \quad \inf _{t \geqq \alpha} F(t)>F(0),
$$

then $I_{F}\left(f_{n}\right) \rightarrow I_{F}(0)$ implies that $\left\{f_{n}\right\}$ converges to 0 in measure.

Proof. First, we shall prove 1). Since we have

$$
\left|I_{F}(f)-I_{F}(0)\right| \leqq \int_{X}|F(|f(x)|)-F(0)| d \mu(x),
$$

we can estimate as follows:

$$
\begin{aligned}
& \left|I_{F}(f)-I_{F}(0)\right| \\
& \quad \leqq \sup _{t \leqq \delta}|F(t)-F(0)|+\sup _{t \geqq \delta}|F(t)-F(0)| \cdot \mu(\{x \in X ;|f(x)| \geqq \delta\}) .
\end{aligned}
$$

Since $F(t)$ is continuous, for any given $\varepsilon>0$, there exists $\delta>0$ such that the first term in (3.7) becomes smaller than $\varepsilon$. Putting $\sup _{t \geqq 0}|F(t)|=M$, we see that for every $f \in U_{\delta, \varepsilon / 2 M}$, the second term in (3.7) becomes smaller than $\varepsilon$. Thus, $f \in U_{\delta, \varepsilon / 2 M}$ implies $\left|I_{F}(f)-I_{F}(0)\right|<2 \varepsilon$, so that 1$)$ has been proved.

Next, we shall prove 2). From the assumption (3.5), we have $F(t) \geqq F(0)$, so that we get

$$
\begin{aligned}
& I_{F}(f)-I_{F}(0) \\
& \quad \geqq \int_{|f(x)| \geqq \alpha}(F(|f(x)|)-F(0)) d \mu(x) \\
& \quad \geqq\left(\inf _{t \geqq \alpha} F(t)-F(0)\right) \cdot \mu(\{x \in X ;|f(x)| \geqq \alpha\}) .
\end{aligned}
$$

Therefore, for any given $\varepsilon>0$ and $\alpha>0, f \in U_{\alpha, \varepsilon}$ can be derived from

$$
I_{F}(f)-I_{F}(0)<\varepsilon\left(\inf _{t \geqq \alpha} F(t)-F(0)\right),
$$

so that 2) has been proved.

Q.E.D.

Thus, if a bounded continuous function $F(t)$ on $[0, \infty)$ satisfies $(3.5),\left\{f_{n}\right\}$ 
converges to 0 in measure if and only if $I_{F}\left(f_{n}\right)$ converges to $I_{F}(0)$. In other words, a fundamental system of neighbourhoods of 0 in the topology of measure convergence is given by

$$
W_{\varepsilon}=\left\{f ; I_{F}(f)<I_{F}(0)+\varepsilon\right\} .
$$

Note that whenever $F(t)$ is monotonically increasing, $F(t)$ satisfies (3.J). Furthermore, if we assume that $F(t)$ is a convex function with $F(0)=0$, then $d(f, g)=I_{F}(f-g)$ becomes a metric on $\mathfrak{F}$, and the topology of measure convergence can be defined by the metric $d$. (Accurately, $d$ is a pseudo-metric on $\mathfrak{F}$, and becomes a metric on $\mathfrak{F} / \mathfrak{M}$, where $\mathfrak{M}$ is given by (3.3).)

Especially, choosing $F(t)=t /(1+t)$, we obtain the metric $d$ given as follows:

$$
d(f, g)=\int_{X} \frac{|f(x)-g(x)|}{1+|f(x)-g(x)|} d \mu(x) .
$$

Proposition 3.1. Let $\mu$ and $v$ be probability measures on $(X, \mathfrak{B})$. If $\nu \lesssim \mu$ ( $v$ is absolutely continuous with respect to $\mu$ ), then the topology of measure convergence corresponding to $\mu$ is stronger than that corresponding to $v$.

Corollary. If $\mu \sim v$, then two topologies of measure convergence are identical with each other.

Proof. Since a neighbourhood of 0 in the topology of measure convergence is given by (3.2), it is sufficient to show that

$$
\forall \varepsilon>0, \quad \exists \delta>0, \quad \mu(B)<\delta \Longrightarrow v(B)<\varepsilon .
$$

We shall prove the contraposition. Assume that

$$
\exists \varepsilon>0, \quad \forall n, \quad \exists B_{n} \in \mathfrak{B}, \quad \mu\left(B_{n}\right)<\frac{1}{2^{n}} \quad \text { and } \quad v\left(B_{n}\right) \geqq \varepsilon .
$$

Then, putting $B=\varlimsup \operatorname{im} B_{n}\left(=\bigcap_{k=1}^{\infty} \bigcup_{n=k}^{\infty} B_{n}\right)$, we get $\mu(B)=0$ and $v(B) \geqq \varepsilon$. This contradicts with $\nu \lesssim \mu$.

Now, we shall return to the case of $\left(E^{\prime}, \mathfrak{B}_{E}\right)$. Every element of $E$, regarded as a function on $E^{\prime}$, is $\mathfrak{B}_{E}$-measurable, so that we can imbed $E$ into $\mathfrak{F}$, where $\mathfrak{\Im}$ is the set of all $\mathfrak{B}_{E}$-measurable real-valued functions on $E^{\prime}$.

Proposition 3.2. For a probability measure on $\left(E^{\prime}, \mathfrak{B}_{E}\right)$, the characteristic topology on $E$ is identical with the restriction of the topology of measure convergence on $E$.

Proof. Since we have 


$$
\begin{aligned}
|1-\chi(\xi)| \leqq & \int_{E^{\prime}}|1-\exp (i(\xi, x))| d \mu(x) \\
\leqq & \int_{E^{\prime}}|1-\cos (\xi, x)| d \mu(x) \\
& +\int_{E^{\prime}}|\sin (\xi, x)| d \mu(x)
\end{aligned}
$$

putting $F_{1}(t)=1-\cos t$ and $F_{2}(t)=|\sin t|$, we have

$$
|1-\chi(\xi)| \leqq I_{F_{1}}(\xi)+I_{F_{2}}(\xi)
$$

Therefore from Lemma 1$), \chi(\xi)$ is continuous with respect to the topology of measure convergence. In other words, the topology of measure convergence is stronger than the characteristic topology on $E$.

Conversely, if $\xi$ belongs to $U_{\varepsilon}$ in (2.5), integrating $1-\chi(\alpha \xi)$ with respect to $\alpha$, we get

$$
\begin{aligned}
\varepsilon & >\left|\frac{1}{2} \int_{-1}^{1}(1-\chi(\alpha \xi)) d \alpha\right| \\
& =\int_{E^{\prime}}\left(1-\frac{\sin (\xi, x)}{(\xi, x)}\right) d \mu(x)
\end{aligned}
$$

However, $F(t)=1-\sin t / t$ is bounded and continuous on $[0, \infty)$ and satisfies the assumption (3.5), so that from Lemma 2), we see that the characteristic topology is stronger than the topology of measure convergence.

Q.E.D.

Corollary. Let $\mu$ and $v$ be probability measures on $\left(E^{\prime}, \mathfrak{B}_{E}\right)$. If $\nu \lesssim \mu$, then the characteristic topology of $\mu$ is stronger than that of $v$. Especially if $v \sim \mu$, then two characteristic topologies are identical with each other.

\section{§4. Proof of our Main Theorems}

For a probability measure $\mu$ on $\left(E^{\prime}, \mathfrak{B}_{E}\right)$, let $E_{\mu}^{*}$ be the topological dual space of $E$ with respect to the characteristic topology $\tau_{\mu}$. (Though $\tau_{\mu}$ is not necessarily Hausdorff nor locally convex, we can define $E_{\mu}^{*}$ as the set of all continuous linear functions in the topology $\tau_{\mu}$.)

For an element $x$ of $E^{\prime}$, the translated measure $\mu_{x}$ is defined by

$$
\mu_{x}(B)=\mu(B-x), \quad \forall B \in \mathfrak{B}_{E} .
$$

Let $T_{\mu}$ be the set of all translations on $E^{\prime}$ under which $\mu$ is quasi-invariant, namely put 


$$
T_{\mu}=\left\{x \in E^{\prime} ; \mu_{x} \sim \mu\right\} .
$$

$T_{\mu}$ forms an additive group, but it is not a vector space in general.

Theorem 4.1. We have $T_{\mu} \subset E_{\mu}^{*}$.

Proof. Suppose that $x \in T_{\mu}$. Since $\mu_{x} \sim \mu$, the characteristic topology of $\mu_{x}$ is identical with that of $\mu$. If we denote the characteristic function of $\mu$ with $\chi(\xi)$, then the characteristic function of $\mu_{x}$ is given by $\exp (i(\xi, x)) \chi(\xi)$. Therefore, in the characteristic topology $\tau_{\mu}$ of $\mu$, the function $\exp (i(\xi, x)) \chi(\xi)$ must be continuous. Since $\chi(\xi)$ is continuous in $\tau_{\mu}$, $\exp (i(\xi, x))$ must be continuous in $\tau_{\mu}$ at every $\xi$ such that $\chi(\xi) \neq 0$, especially at $\xi=0$. Namely, for some neighbourhood $U_{\varepsilon}$ in (2.5), we have

$$
\xi \in U_{\varepsilon} \Longrightarrow|1-\exp (i(\xi, x))|<1 .
$$

However, $\xi \in U_{\varepsilon}$ implies $\alpha \xi \in U_{\varepsilon}$ for $|\alpha| \leqq 1$, so we have $|1-\exp (i \alpha(\xi, x))|<1$ for $|\alpha| \leqq 1$, hence we have $|(\xi, x)|<\pi / 3$.

Thus, $|(\xi, x)|$ is bounded on $U_{\varepsilon}$. This means that $x$ is continuous in $\tau_{\mu}$, namely $x \in E_{\mu}^{*}$.

This holds for every $x \in T_{\mu}$, so we have $T_{\mu} \subset E_{\mu}^{*}$.

Q.E.D.

Corollary. If $E$ is a topological vector space in some topology $\tau$, and if $\chi(\xi)$ is continuous in $\tau$, then we have $T_{\mu} \subset E_{\tau}^{*}$, where $E_{\tau}^{*}$ is the topological dual space of $E$ with respect to $\tau$.

Proof. Since $\chi$ is continuous in $\tau, \tau_{\mu}$ is weaker than $\tau$, so that we have $E_{\mu}^{*} \subset E_{\tau}^{*}$. Therefore, we get the corollary from Theorem 4.1. $\quad$ Q.E.D.

This corollary enables us to tell about the quasi-invariance of a measure in terms of the continuity of the characteristic function. For instance, for a measure on $\boldsymbol{R}^{\infty}$, if the characteristic function is continuous in the $\left(l^{2}\right)$-norm on $\boldsymbol{R}_{0}^{\infty}$, then we must have $T_{\mu} \subset\left(l^{2}\right)$.

For a subset $A$ of $E$, we shall put

$$
\|x\|_{A}=\sup _{\xi \in A}|(\xi, x)|, \quad \forall x \in E^{\prime} .
$$

Similarly for a subset $A^{\prime}$ of $E^{\prime}$, we shall put

$$
\|\xi\|_{A^{\prime}}=\sup _{x \in A^{\prime}}|(\xi, x)|, \quad \forall \xi \in E .
$$

These values may be $\infty$. The set of all $x$ such that $\|x\|_{A}<\infty$ forms a linear subspace of $E^{\prime}$, and $\|\cdot\|_{A}$ becomes a semi-norm on this space. Similar arguments hold for $\|\cdot\|_{A^{\prime}}$. 
Theorem 4.2. Let $Y$ be a linear subspace of $E^{\prime}$. Suppose that $Y$ is a complete metrizable topological vector space with respect to a topology stronger than the weak topology on $E^{\prime}$. If $Y \subset T_{\mu}$, then for some neighbourhood $V$ of 0 in $Y,\|\xi\|_{V}$ is finite for all $\xi \in E$, and the semi-normed topology defined by $\|\cdot\|_{V}$ is weaker than the characteristic topology $\tau_{\mu}$.

Proof. For a neighbourhood $U_{\varepsilon}$ of 0 in the characteristic topology, let $A_{\varepsilon}$ be its polar set, namely put

$$
A_{\varepsilon}=\left\{x \in E^{\prime} ;|(\xi, x)| \leqq 1 \text { for } \xi \in U_{\varepsilon}\right\} .
$$

Then, $A_{\varepsilon}$ is evidently a weakly closed subset of $E^{\prime}$, so that $A_{\varepsilon} \cap Y$ is a closed subset of $Y$.

Since $\left\{U_{1 / n}\right\}_{n=1,2, \ldots}$ is a fundamental system of neighbourhoods of 0 in $\tau_{\mu}, x \in E_{\mu}^{*}$ is equivalent with $x \in A_{1 / n}$ for some $n$, namely we have

$$
\bigcup_{n=1}^{\infty} A_{1 / n}=E_{\mu}^{*} \supset T_{\mu} \supset Y .
$$

Thus, we get $\bigcup_{n=1}^{\infty}\left(A_{1 / n} \cap Y\right)=Y$. Since $Y$ is a complete metric space, from Baire's category theorem, some $A_{1 / n} \cap Y$ has an inner point in $Y$. Namely, we have

$$
\begin{aligned}
& \left.\exists y_{0} \in Y, \quad \exists V \text { (neighbourhood of } 0 \text { in } Y\right), \\
& y_{0}+V \subset A_{1 / n} \cap Y .
\end{aligned}
$$

We shall show that this $V$ is a requested one in our theorem. If $y \in V$ and $\xi \in U_{1 / n}$, then we have

$$
|(\xi, y)| \leqq\left|\left(\xi, y+y_{0}\right)\right|+\left|\left(\xi, y_{0}\right)\right| \leqq 2 .
$$

Therefore $\xi \in U_{1 / n}$ implies $\|\xi\|_{V} \leqq 2$. Hence, $\xi \in \alpha U_{1 / n}$ implies $\|\xi\|_{V} \leqq 2 \alpha$. This shows that $\|\xi\|_{V}$ is finite for all $\xi \in E$ and that the topology defined by $\|\cdot\|_{V}$ is weaker than $\tau_{\mu}$.

Q.E.D.

Theorem 4.3. Suppose that $E$ is a locally convex vector space with respect to a topology $\tau$. If $E_{\tau}^{*} \subset T_{\mu}$, then $\tau$ is weaker than $\tau_{\mu}$.

Proof. Let $U$ be a convex symmetric neighbourhood of 0 in $\tau$. Defining $\|\cdot\|_{U}$ on $E^{\prime}$ by (4.4), we shall put

$$
E_{U}^{*}=\left\{x \in E^{\prime} ;\|x\|_{U}<\infty\right\} .
$$

Evidently we have $E_{U}^{*} \subset E_{\tau}^{*}$, so that we have $E_{U}^{*} \subset T_{\mu}$. On the other hand, 
$E_{U}^{*}$ is a Banach space with respect to $\|\cdot\|_{U}$, and its topology is stronger than the weak topology on $E^{\prime}$. Therefore, there exists a neighbourhood $V$ of 0 mentioned in Theorem 4.2. Since $E_{U}^{*}$ is a normed space, we can suppose that $V$ is the unit ball in $E_{U}^{*}$. Then, from Theorem 4.2 , the topology defined by $\|\cdot\|_{V}$ is weaker than $\tau_{\mu}$, namely the polar set

$$
B=\left\{\xi \in E ;\|\xi\|_{V} \leqq 1\right\}
$$

contains a neighbourhood of 0 in $\tau_{\mu}$. However, since $V$ is the polar set of $U, B$ is the bipolar set of $U$. Therefore, remembering that $U$ is a convex symmetric set, we have $B=\bar{U}$ ( $=$ the closure of $U$ ). Thus, $\bar{U}$ contains a neighbourhood of 0 in $\tau_{\mu}$.

This fact holds for every convex symmetric neighbourhood $U$ in $\tau$. Since the topology of a topological group is regular, this means that $\tau$ is weaker than $\tau_{\mu}$.

Q.E.D.

Corollary. Suppose that $E$ is a locally convex topological vector space with respect to a topology $\tau$. For a probability measure $\mu$ on $\left(E^{\prime}, \mathfrak{B}_{E}\right)$, if $\mu$ is $E_{\tau}^{*}$-quasi-invariant and if the characteristic function $\chi$ is continuous in $\tau$, then we have $T_{\mu}=E_{\tau}^{*}$ and $\tau=\tau_{\mu}$.

Proof. Since $\mu$ is $E_{\tau}^{*}$-quasi-invariant, we have $T_{\mu} \supset E_{\tau}^{*}$ and $\tau$ is weaker than $\tau_{\mu}$

Since $\chi$ is continuous in $\tau$, we have $T_{\mu} \subset E_{\tau}^{*}$ and $\tau_{\mu}$ is weaker than $\tau$.

Q.E.D.

For instance, for a Borel measure $\mu$ on $\mathbb{R}^{\infty}$, if $\mu$ is $\left(l^{2}\right)$-quasi-invariant and if the characteristic function is continuous in the $\left(l^{2}\right)$-norm, then we have $T_{\mu}=$ $\left(l^{2}\right)$ and the characteristic topology $\tau_{\mu}$ is identical with the topology of $\left(l^{2}\right)$.

Assume that $T_{\mu}=E_{\mu}^{*}$, then $\mu$ is $E_{\mu}^{*}$-quasi-invariant and $\chi$ is continuous in $\tau_{\mu}$. The corollary insists that such a situation (that $\mu$ is $E_{\tau}^{*}$-quasi-invariant and $\chi$ is continuous in $\tau$ ) occurs only for the characteristic topology $\tau_{\mu}$. Furthermore, such a situation occurs for $\tau_{\mu}$ if and only if $T_{\mu}=E_{\mu}^{*}$.

\section{§5. Totally Boundedness}

As applications of the theorems in Section 4, in this and the following sections, we shall prove the results 1 and 2 mentioned in Introduction.

First we shall prove: 
Theorem 5.1. Let $X$ be a separable and metrizable locally convex vector space, and $Y(\subset X)$ be a complete metrizable topological vector space imbedded continuously in $X$. If some Borel measure on $X$ is $Y$-quasi-invariant, then $Y$ has a neighbourhood of 0 which is totally bounded in $X$.

Proof. Let $X^{*}$ be the topological dual space of $X$. Then, $X$ can be imbedded into $\left(X^{*}\right)^{\prime}$, the algebraical dual space of $X^{*}$. We can define the measurable space $\left(\left(X^{*}\right)^{\prime}, \mathfrak{B}_{X^{*}}\right)$ as in Section 2 , replacing $E$ with $X^{*}$. Denoting the Borel algebra of $X$ with $\mathfrak{B}$, we shall first prove:

$$
\mathfrak{B}=\mathfrak{B}_{X^{*}} \cap X .
$$

For every $\xi \in X^{*}$, since $(\xi, x)$ is continuous on $X$, it is $\mathfrak{B}$-measurable, so that we have $\mathfrak{B} \supset \mathfrak{B}_{X^{*}} \cap X$.

Conversely, we shall show that every open set of $X$ belongs to $\mathfrak{B}_{X^{*}} \cap X$. Since $X$ is separable and metrizable, every open set $O$ of $X$ satisfies Lindelöf's property (that is, an open covering of $O$ contains necessarily a countable covering of $O$ ). Therefore, there exist a sequence of points $\left\{x_{n}\right\}$ and a sequence of convex symmetric neighbourhoods $\left\{U_{n}\right\}$ of 0 such that

$$
O=\bigcup_{n=1}^{\infty}\left(x_{n}+U_{n}\right) \text {. }
$$

We shall show $U_{n} \in \mathfrak{B}_{X^{*}} \cap X$. We can suppose that $U_{n}$ is a closed neighbourhood. Then, $U_{n}$ is the bipolar set of itself, namely we have

where

$$
\begin{array}{lll}
U_{n}=\{x \in X ;|(\xi, x)| \leqq 1 & \text { for every } & \left.\xi \in A_{n}\right\}, \\
A_{n}=\left\{\xi \in X^{*} ;|(\xi, x)| \leqq 1\right. & \text { for every } & \left.x \in U_{n}\right\}
\end{array}
$$

Thus, $U_{n}$ is a weakly closed set. However, a weakly open set of $X$ belongs to $\mathfrak{B}_{X^{*}} \cap X$, because from Lindelöf's property it can be written as a countable union of weak neighbourhoods, which belong evidently to $\mathfrak{B}_{X^{*}} \cap X$.

Hence $U_{n}$, so the given $O$ also, belongs to $\mathfrak{B}_{X^{*}} \cap X$. This completes the proof of (5.1).

From (5.1), a Borel measure on $X$ can be identified with a measure on $\left(\left(X^{*}\right)^{\prime}, \mathfrak{B}_{X^{*}}\right)$. Since $Y$ is continuously imbedded in $X$, it is also imbedded in $\left(X^{*}\right)^{\prime}$, and the topology of $Y$ is stronger than the weak topology of $\left(X^{*}\right)^{\prime}$. Therefore, if the measure $\mu$ is $Y$-quasi-invariant, then from Theorem 4.2, there exists a neighbourhood $V$ of 0 in $Y$ such that the semi-normed topology defined by $\|\cdot\|_{V}$ is weaker than the characteristic topology $\tau_{\mu}$. Then, the unit ball in $\|\cdot\|_{V}$ contains a neighbourhood of 0 in $\tau_{\mu}$. 
On the other hand, since the measure $\mu$ lies on $X$, for any given $\varepsilon>0$ there exists a totally bounded set $B$ such that $\mu(B)>1-\varepsilon$. (The Borel measure $\mu$ on $X$ can be identified with a Borel measure on $\bar{X}$, the completion of $X$. Since $\bar{X}$ is complete, metrizable and separable, every Borel measure on $\bar{X}$ lies on a countable union of compact sets. Cf. Parthasarathy [4].)

Considering the semi-norm $\|\cdot\|_{B}$ on $X^{*}$, we have

$$
\begin{aligned}
|1-\chi(\xi)| & \leqq \int_{X}|1-\exp (i(\xi, x))| d \mu(x) \\
& \leqq \int_{B}|(\xi, x)| d \mu(x)+2 \mu\left(B^{\mathbf{C}}\right) \\
& \leqq\|\xi\|_{B}+2 \varepsilon
\end{aligned}
$$

so that we have

$$
\|\xi\|_{B}<\varepsilon \Longrightarrow|1-\chi(\xi)|<3 \varepsilon .
$$

Since $\|\xi\|_{B}<\varepsilon$ is equivalent with $\|\xi\|_{B / \varepsilon}<1$, (5.4) implies that each neighbour hood of 0 in $\tau_{\mu}$ contains the unit ball in $\|\cdot\|_{A}$ where $A$ is some totally bounded set of $X$.

Thus, the unit ball in $\|\cdot\|_{V}$ contains the unit ball in $\|\cdot\|_{A}$. Therefore we have

$$
V \subset C(A),
$$

where $C(A)$ is the convex closed hull of $A$. Since $A$ is totally bounded, $C(A)$, hence $V$ also is totally bounded in $X$.

Q.E.D.

Corollary. Let $X$ be a complete, metrizable and separable locally convex vector space. (For instance, suppose that $X$ is a separable Banach space or a separable Fréchet space.) Let $Y$ be a closed subspace of $X$. If some Borel measure on $X$ is Y-quasi-invariant, then $Y$ must be finite dimensional. In other words, $T_{\mu}$ never contains an infinite dimensional closed subspace.

Proof. Since $Y$ is closed, $Y$ becomes a complete metrizable topological vector space with respect to the induced topology from $X$. Therefore from Theorem 5.1, some neighbourhood of 0 in $Y$ must be totally bounded in $X$, hence in $Y$. This means that $Y$ is locally totally bounded. This is impossible unless $Y$ is finite dimensional.

Q.E.D.

Example. Let $\left(l^{p}\right)$ be the set of all $p$-th power summable sequences:

$$
\left(l^{p}\right)=\left\{x=\left(x_{n}\right) ; \sum_{n=1}^{\infty}\left|x_{n}\right|^{p}<\infty\right\} .
$$


$\left(l^{p}\right)$ is monotonically increasing, namely $p^{\prime}<p$ implies $\left(l^{p^{\prime}}\right) \subset\left(l^{p}\right)$. However, if $p<\infty$, no Borel measure on $\left(l^{p}\right)$ is $\left(l^{p^{\prime}}\right)$-quasi-invariant, because the unit ball in $\left(l^{p^{\prime}}\right)$ is not totally bounded in $\left(l^{p}\right)$. (Putting $e_{k}=\left(\delta_{l: n}\right)_{n=1,2, \ldots} \in\left(l^{p^{\prime}}\right)$, we have $\left\|e_{k}\right\|_{p^{\prime}}=1$ and $\left\|e_{k}-e_{j}\right\|_{p} \geqq 1$ for $k \neq j$.)

For $p=\infty$, though $\left(l^{\infty}\right)$ is a Banach space, it is not separable, so Theorem 5.1 can not be applied. In fact, some Borel measure on $\boldsymbol{R}^{\infty}$ is $\left(l^{1}\right)$-quasi-invariant and lies on $\left(l^{\infty}\right)$ (cf. [5]). However the space $\left(l_{0}^{\infty}\right)$, the set of all sequences such that $\lim _{n \rightarrow \infty} x_{n}=0$, is separable in $\|\cdot\|_{\infty}$, so that Theorem 5.1 can be applied, thus no Borel measure on $\left(l_{0}^{\infty}\right)$ is $\left(l^{1}\right)$-quasi-invariant.

\section{§6. Hilbert-Schmidt Imbedding}

In this section, we shall prove the result 2 in Introduction. Namely we shall prove:

Theorem 6.1. Let $X$ be a Hilbert space, and $Y(\subset X)$ be another Hilbert space imbedded continuously in $X$. There exists a Y-quasi-invariant measure on $\left(X, \mathfrak{B}_{X^{*}} \cap X\right)$ if and only if the canonical imbedding $Y \rightarrow X$ is of the Hilbert-Schmidt type. Especially, $Y$ must be separable even if $X$ is not.

Proof. Suppose that the topology of $X$ is defined by the inner product $(,)_{X}$, and the topology of $Y$ is defined by $(,)_{Y}$. Consider the gaussian measure $g$ corresponding to $(,)_{Y}$, then $g$ is $Y$-quasi-invariant and lies on $X$, if $(,)_{X}$ is of the Hilbert-Schmidt type with respect to $(,)_{Y}$ on $Y$ (cf. [6]). Thus, the sufficiency part of Theorem 6.1 has been proved.

We shall prove the necessity part of Theorem 6.1. Let $(,)_{X}^{\prime}$ and $(,)_{Y}^{\prime}$ be the dual inner products of $(,)_{X}$ and $(,)_{Y}$ respectively defined on $X^{*}$, the topological dual space of $X$. Imbedding $X$ into $\left(X^{*}\right)^{\prime}$, we can suppose that a measure $\mu$ on $\left(\left(X^{*}\right)^{\prime}, \mathfrak{B}_{X^{*}}\right)$ is $Y$-quasi-invariant and lies on $X$. Since the topology of $Y$ is stronger than the weak topology of $\left(X^{*}\right)^{\prime}$, from Theorem 4.2 we see that the topology defined by $(,)_{Y}^{\prime}$ is weaker than the characteristic topology $\tau_{\mu}$.

On the other hand, since $\mu$ lies on $X$, the characteristic function is continuous in Sazonov topology of $(,)_{X}^{\prime}$, hence Sazonov topology is stronger than $\tau_{\mu}$. (Cf. [2], Sazonov topology is the topology defined by the family of all inner products which are of the Hilbert-Schmidt type with respect to $(,)_{X}^{\prime}$.) Thus, the topology defined by $(,)_{Y}^{\prime}$ is weaker than Sazonov topology, namely $(,)_{Y}^{\prime}$ 
is continuous in Sazonov topology. This means that $(,)_{Y}^{\prime}$ is Hilbert-Schmidt with respect to $(,)_{X}^{\prime}$. Therefore, on the space $Y$, the dual inner product $(,)_{X}$ must be Hilbert-Schmidt with respect to $(,)_{Y}$. This implies that the canonical imbedding $Y \rightarrow X$ is of the Hilbert-Schmidt type, so that the proof has been completed.

Q.E.D.

This theorem can be generalized to the case that $Y$ is a complete metrizable topological vector space.

Proposition 6.1. Let $X$ be a Hilberi space, $Y(\subset X)$ be a complete metrizable topological vector space imbedded continuously in $X$. Then, there exists a $Y$-quasi-invariunt measure on $\left(X, \mathfrak{B}_{X^{*}} \cap X\right)$ if and only if $(,)_{X}$ is of the HilbertSchmidt type with respect to some continuous inner product $(,)_{H}$ on $Y$.

Proof. The sufficiency part can be proved by considering the gaussian measure corresponding to $(,)_{H}$.

For the proof of the necessity part, similarly with the proof of Theorem 6.1 , we see that there exists a neighbourhood $V$ of 0 in $Y$ such that $\|\cdot\|_{V}$ is continuous in $\tau_{\mu}$. Since $\tau_{\mu}$ is weaker than Sazonov topology of $(,)_{X}^{\prime}$, this implies that $\|\cdot\|_{V}$ is continuous in Sazonov topology, therefore we have $\|\xi\|_{V} \leqq\|\xi\|_{H}^{\prime}$ for some inner product $(,)_{H}^{\prime}$ which is of the Hilbert-Schmidt type with respect to $(,)_{X}^{\prime}$.

On the space $Y$, considering the dual inner product $(,)_{H}$ of $(,)_{H}^{\prime}$, we see that $(,)_{X}$ is of the Hilbert-Schmidt type with respect to $(,)_{H}$ and that $(,)_{H}$ $\begin{array}{ll}\text { is continuous in } Y \text {. } & \text { Q.E.D. }\end{array}$

The reason why we discuss a measure on a Hilbert space is that we know exactly the continuity of the characteristic function by Sazonov's theorem.

Even if $X$ is not a Hilbert space, we can estimate $T_{\mu}$ by similar discussions, if we know some results on the continuity of the characteristic function. For instance, we can prove:

Proposition 6.2. For a sequence $a=\left(a_{n}\right)$ of positive numbers, we shall define the space $\left(l^{p}\right)_{a}$ as follows:

$$
\left(l^{p}\right)_{a}=\left\{x=\left(x_{n}\right) ; \sum_{n=1}^{\infty} a_{n}\left|x_{n}\right|^{p}<\infty\right\} .
$$

(If $a_{n} \equiv 1$, then we get the usual $\left(l^{p}\right)$.)

For $1 \leqq p<\infty$, if an ( $\left.\left.\right|^{p^{\prime}}\right)$-quasi-invariant Borel measure exists on $\left(l^{p}\right)_{a}$, then we must have $\sum_{n=1}^{\infty} a_{n}<\infty$. 
Remark. Considering the case $a_{n} \equiv 1$, we obtain again the result of the example in Section 5.

Proof. Suppose that a Borel measure $\mu$ on $\left(l^{p}\right)_{a}$ is $\left(l^{p^{\prime}}\right)$-quasi-invariant. From Theorem 4.2, if we denote the unit ball of $\left(l^{p^{\prime}}\right)$ with $V$, the semi-norm $\|\cdot\|_{V}$ is continuous in the characteristic topology $\tau_{\mu}$ on $\boldsymbol{R}_{0}^{\infty}$.

Denoting the unit ball of $\left(l^{p}\right)_{a}$ with $B$, we have

$$
\lim _{n \rightarrow \infty} \mu(n B)=1 \text {. }
$$

On the other hand, we have

$$
\begin{aligned}
|1-\chi(\xi)| & \leqq \int_{\boldsymbol{R}^{\infty}}|1-\exp (i(\xi, x))| d \mu(x) \\
& \leqq\|\xi\|_{n}+2 \mu\left(n B^{\mathbf{C}}\right)
\end{aligned}
$$

where

$$
\|\xi\|_{n}=\int_{n B}|(\xi, x)| d \mu(x) .
$$

Therefore, the topology defined by the family of semi-norms $\|\cdot\|_{n}$ is stronger than $\tau_{\mu}$. Thus, the semi-norm $\|\cdot\|_{V}$ is continuous in the topology defined by $\left\{\|\cdot\|_{n}\right\}$, so that we have

$$
\exists C>0, \quad \exists n, \quad\|\xi\|_{V} \leqq C\|\xi\|_{n} .
$$

Putting $e_{k}=\left(\delta_{k n}\right)_{n=1,2, \ldots} \in \boldsymbol{R}_{0}^{\infty}$, we have

$$
\left|\left(e_{k}, x\right)\right|=\left|x_{k}\right|,
$$

so that $x \in V$ implies $\left|\left(e_{k}, x\right)\right| \leqq 1$, thus we have $\left\|e_{k}\right\|_{V}=1$. Substituting this into (6.4), we get $1 \leqq C\left\|e_{k}\right\|_{n}$, namely we get

$$
1 \leqq C \int_{n B}\left|x_{k}\right| d \mu(x)
$$

Taking the $p$-th power of the both hand sides, and applying Hölder's inequality, we get

$$
1 \leqq C^{p} \int_{n B}\left|x_{k}\right|^{p} d \mu(x)
$$

Multiplying $a_{k}$ and summing up the both hand sides with $k$, we have

$$
\sum_{k=1}^{\infty} a_{k} \leqq C^{p} \int_{n B} \sum_{k=1}^{\infty} a_{k}\left|x_{k}\right|^{p} d \mu(x),
$$


but the integrand being $\leqq n^{p}$, we get $\sum_{k=1}^{\infty} a_{k} \leqq C^{p} n^{p}<\infty$. This completes the proof of Proposition 6.2.

Q.E.D.

\section{References}

[1] Minlos, R. A., Generalized random processes and their extension to measures, Trudy Moskov. Mat. Obsc., 8 (1959), 497-518.

[2] Sazonov, V., A remark on characteristic functionals, Theory Prob. Appl., 3 (1958), 188-192.

[3] Xia, Dao-Xing, Measures and integration theory on infinite dimensional spaces, Academic Press, 1972.

[4] Parthasarathy, K. R., Probability measures on metric spaces, Academic Press, 1967.

[5] Yamasaki, Y., Translationally invariant measure on the infinite dimensional vector space, Publ. RIMS, Kyoto Univ., 16 (1980), 693-720.

[6] Umemura, Y., Measures on infinite dimensional vector spaces, Publ. RIMS, Kyoto Univ., 1 (1965), 1-47.

Added in proof: Note for the last statement in Section 4: Under the assumption that $\tau_{\mu}$ is locally convex, $T_{\mu}=E_{\mu}^{*}$ occurs only if $\left(E, \tau_{\mu}\right)$ is a Hilbert space. This result was obtained recently by $\mathrm{H}$. Shimomura, and will be published in the near future. 
